MATEC Web of Conferences 51, 01002 (2016)

DOI: $10.1051 /$ matecconf/20165101002

(C) Owned by the authors, published by EDP Sciences, 2016

\title{
Rollover Dynamics of a Narrow Tilting Three-Wheeled Vehicle
}

\author{
Jubin Antony $\mathrm{J}^{1, \mathrm{a}}$ and Jayabal $\mathrm{K}^{1, \mathrm{~b}}$ \\ ${ }^{1}$ Indian Institute of Information Technology Design and Manufacturing, Kancheepuram, Tamil Nadu, India
}

\begin{abstract}
A narrow-track vehicle possesses many advantages like fuel efficiency and reduced road footprint among others. But the main drawback of this model stems from the fact that they tend to rollover more easily due to their narrow base area. In order to overcome this rollover instability, these vehicles are made to tilt to obtain increased virtual base area. The focus of this paper is to analyse the minimum lean angle necessary for a narrow three-wheeled vehicle under lateral acceleration to negotiate a curve safely. In addition, the influence of the horizontal positions of the centre of mass of the vehicle over the rollover speeds is studied.
\end{abstract}

\section{Introduction}

Due to sharp increase in the cost of fuel and environmental pollution during the past ten years, there is an increasing demand for producing an energy efficient passenger vehicle. In the existing four wheeled designs, the scope for further fuel efficiency is limited. On the other hand, the two wheeled vehicles have a better fuel efficiency for single users but in this model the passenger is exposed to bad weather and generally has a very less safety factor. Therefore, the three-wheeled vehicle design would be a better scope for producing a fuel efficient model, without sacrificing the safety and comfort of the passenger.

Since the three-wheeled vehicle has a reduced base as compared to an equivalent four-wheeled vehicle, it has a higher probability of tipping over while cornering a turn. Huston [1] has proved it by comparing a non-tilting three wheeled vehicle with a standard four-wheeled vehicle. In order to prevent the rollover, it was suggested by Karnopp [2] that the vehicle should lean like a motorcycle.Subsequently, Hibbard and Karnoppdiscussed the dynamics of a narrow tilting vehicle [3] and the method to control the leaning angle of tilting vehicles [4].

Among tilting three-wheeled vehicles, Palash [5] carried on with the analysis of a tilting tadpole model and their advantages.Berote [6] described about the dynamics of a tilting delta model and the effect ofhydraulics have on the system. Despite the future potential of this kind of vehicles, not too many research articles are found in the literature on the rollover dynamics of tilting of three-wheeled vehicles.

In this paper, the effect of leaning on the rollover speed is analysed and comparisons of rollover speeds are discussed between two types of three-wheeled vehicles, the tadpole and delta configurations; the former one will have two wheels at the front axle and one at the rear whereas the later one will have vice-versa.

${ }^{a}$ Correspondingauthor : mdm12b011@iiitdm.ac.in

bjayabal@iiitdm.ac.in 


\section{MATEC Web of Conferences}

\section{Nomenclature}

$l_{1} \quad$ Longitudinal distance from centre of mass to front axle

$l_{2} \quad$ Longitudinal distance from centre of mass to rear axle

$\mathrm{L} \quad$ Longitudinal distance from rear axle to front axle

W Weight of the vehicle

$\mathrm{h} \quad$ Height of centre of mass of the vehicle without leaning

$\mathrm{R} \quad$ Radius of curvature of the turn

$\mathrm{V}_{\mathrm{y}} \quad$ Longitudinal velocity along y-direction

$\mathrm{a}_{\mathrm{y}} \quad$ Longitudinal acceleration along y-direction

E Inertia force vector due to lateral acceleration

A Inertia force vector due to longitudinal acceleration

D Inertia force vector due to longitudinal deceleration

$\mathbf{Z} \quad$ Resultant force acting on the centre of mass

$\theta \quad$ Angle of leaning of the vehicle

$\varnothing \quad$ Angle between $\mathbf{Z}$ and $\mathrm{x}$-axis

$\gamma \quad$ Angle between $\mathbf{E}$ and $\mathrm{x}$-axis

$\alpha, \beta, \alpha_{d}, \beta_{d} \quad$ Angle between $\mathbf{Z}$ and the normal to TT axis passing through centre of mass

$\delta$

Angle between the normal to TT axis passing through centre of mass and $\mathrm{x}$-axis

\section{Rollover Stability}

Rollover stability is the ability of the vehicle to resist tipping over under the action of lateral body force. The lateral force may be due to the centrifugal force while cornering or due to the air resistance. For a good vehicle design, rollover stability should be as high as possible.

In all figures appearing in the article, the axis of tipping is denoted by TT. To ensure rollover stability, the clockwise moment about the TT axis, as viewed from the rear end, should be less than zero.

$$
\sum M_{T T}<0
$$

\subsection{Three-wheeled Tadpole Model}

In this section, a three-wheeled tadpole configuration is considered. It is assumed that when the vehicle is leaned, the centre of mass of the vehicle is displaced by an angle $\theta$ with respect to the point $\mathrm{P}$ in the ground plane GG as shown in Figure 1. Let $\mathrm{X}$ be the perpendicular distance between the TT axis and the centre of mass as shown in Figure 2. The magnitude of $\mathbf{Z}, \mathbf{A}$ and $\mathbf{E}$ are given by

$$
\begin{gathered}
Z=|\mathbf{Z}|=|E \cos \gamma \hat{\imath}+(E \sin \gamma-A) \hat{\jmath}|=\left[A^{2}+E^{2}-2 A E \sin \gamma\right]^{0.5} \\
A=|\mathbf{A}|=\frac{W a_{y}}{g} \\
E=|\mathbf{E}|=\frac{W}{g} \frac{V_{y}{ }^{2}}{R}
\end{gathered}
$$

The moment about the axis TT will be

$$
\sum M_{T T}=-W X+Z h \cos \alpha \cos \theta<0
$$

From Figure 4, it can be shown using trigonometric rules that

$$
X=(c \tan \delta) \cos \delta=\left(l_{2}+\frac{2 h L \sin \theta}{b}\right) \sin \delta
$$




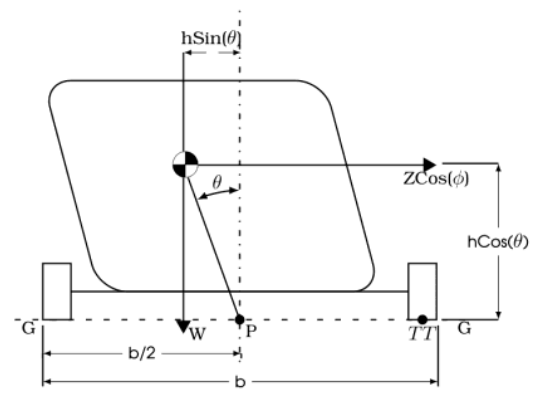

Figure 1.Front view of three wheeled tadpole model

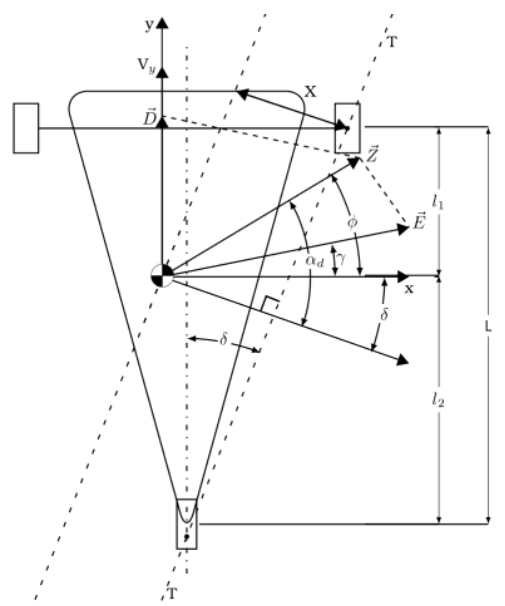

Figure 3. Top view of three wheeled tadpole model while braking

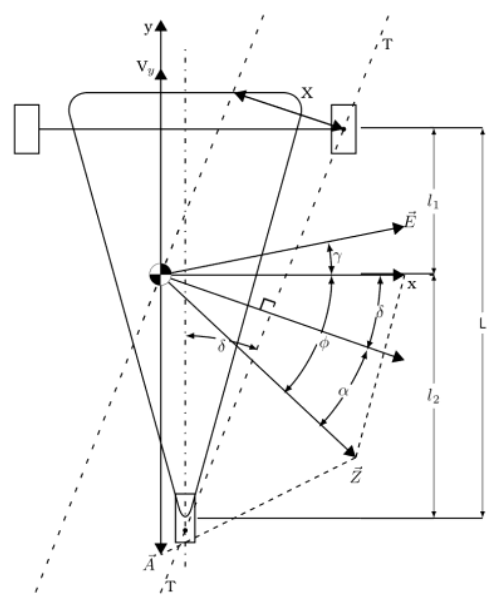

Figure 2.Top view of three wheeled tadpole model while accelerating

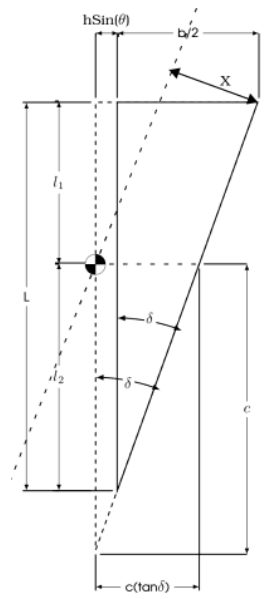

Figure 4. Top view of the centre of mass displaced area

\subsubsection{Cornering a curve with a constant velocity}

If the vehicle is negotiating a turn with a constant velocity, then the longitudinal acceleration will be zero, $\mathrm{a}_{y}=0$ (i.e. $\mathbf{Z}=\mathbf{E}$ ) and $\alpha=\gamma+\delta$. Thus, eq. (5) will be

$$
\sum M_{T T}=-W\left(l_{2}+\frac{2 h L \sin \theta}{b}\right) \sin \delta+\frac{W}{g} \frac{V_{y}{ }^{2}}{R} h \cos \alpha \cos \theta<0
$$

When eq. (7) is equated to zero, the rollover speed is determined as

$$
V_{r o}=\sqrt{\frac{R g b\left(l_{2}+\frac{2 h L \sin \theta}{b}\right)}{2 h L \cos \alpha \cos \theta}}
$$




\subsubsection{Cornering a curve with an acceleration}

In this case, the vehicle is cornering a turn with a longitudinal acceleration (i.e. $A \neq 0$ ) and also $\mathbf{Z}$ will have component of Aas shown in eq. (2). Thus, the rollover stability condition will be

where

$$
\begin{gathered}
\sum M_{T T}=-W\left(l_{2}+\frac{2 h L \sin \theta}{b}\right) \sin \delta+Z h \cos \alpha \cos \theta<0 \\
Z \cos \alpha<\frac{W\left(l_{2} b+2 h L \sin \theta\right) \sin \delta}{b h \cos \theta}
\end{gathered}
$$

Eq. (11) and (12) can be geometrically calculated from Figure 2.

\subsubsection{Cornering a curve with braking}

While braking on a turn, Dwill be acting along the positive y-axis as shown in Figure 3. Similarly like the above condition, the rollover stability condition can be represented as

$$
Z \cos \alpha_{d}<\frac{W\left(l_{2} b+2 h L \sin \theta\right) \sin \delta}{b h \cos \theta}
$$

In eq. (13), the value of $\alpha_{d},\left(\alpha_{d}=\phi+\delta\right)$ can be geometrically found out from Figure 3. Eq. (10) and (13) should be solved numerically in order to obtain the rollover speeds.

\subsection{Three-wheeled DeltaModel}

Similarly like the tadpole model, the three wheeled delta model, as shown in Figures 5 and 6, can also be solved to find rollover speeds.

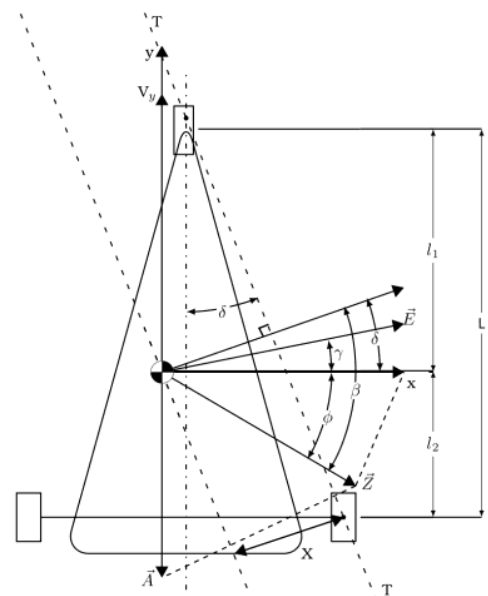

Figure 5. Top view of three wheeled delta model while accelerating.

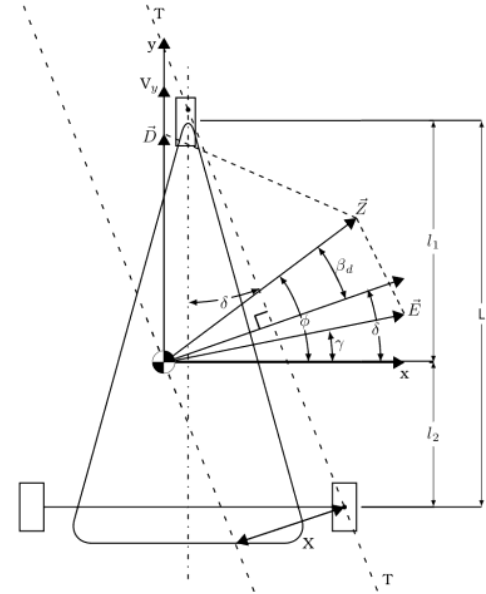

Figure 6.Top view of three wheeled delta model while braking. 
The rollover speeds for various cases are given as

$$
\begin{gathered}
V_{r o=} \sqrt{\frac{R g b\left(l_{1}+\frac{2 h L \sin \theta}{b}\right)}{2 h L \cos \alpha \cos \theta}} \\
Z \operatorname{Cos}(\beta=\phi+\delta)=\frac{W\left(l_{1} b+2 h L \sin \theta\right) \sin \delta}{b h \cos \theta} \\
Z \operatorname{Cos}\left(\beta_{d}=\phi-\delta\right)=\frac{W\left(l_{1} b+2 h L \sin \theta\right) \sin \delta}{b h \cos \theta}
\end{gathered}
$$

Eq. (14), (15) and (16) provide the rollover speed equations, respectively, when the vehicle is moving at a constant speed, during acceleration and during deceleration. Eq. (15) and (16) should be solved numerically to find the rollover speeds.

\section{Simulation}

For simulation, the vehicle model taken had a mass of $\mathrm{m}=400 \mathrm{Kg}$, length of $\mathrm{L}=2 \mathrm{~m}$, a base length of $b=0.6 \mathrm{~m}$ and a centre of mass height of $\mathrm{h}=0.6 \mathrm{~m}$. It is assumed to negotiate a curve of radius, $\mathrm{R}=100 \mathrm{~m}$ with the acceleration magnitude of $(0.25 \mathrm{~g}) \mathrm{m} / \mathrm{s}^{2}$. To illustrate the importance of the horizontal position of the centre of mass, various positions of centre of mass is taken. Also, the graphs are plotted for various lean angles to know the significance of leaning; refer Figures 7 to 12.

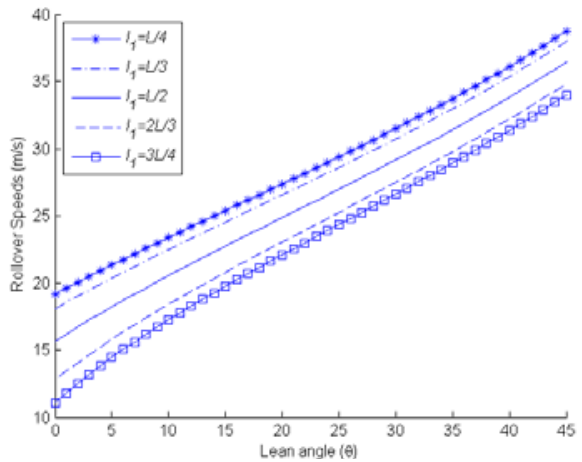

Figure 7. Three-wheeled Tadpole vehicle with no acceleration

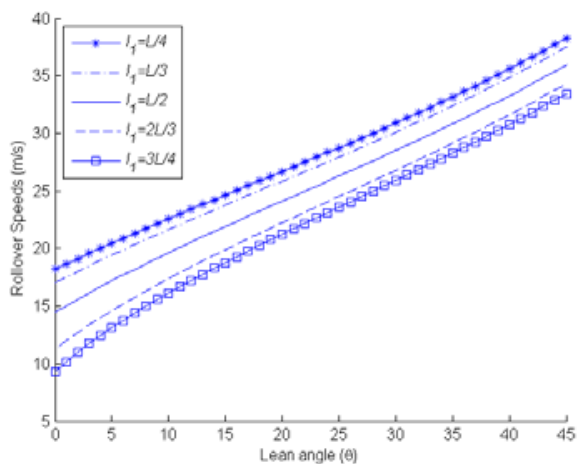

Figure 9. Three-wheeled Tadpole vehicle with acceleration

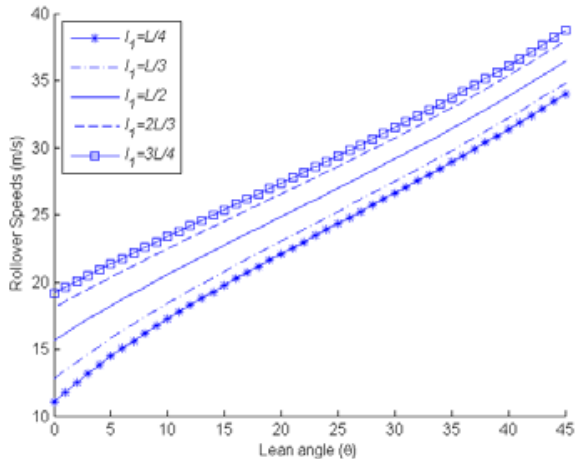

Figure 8. Three-wheeled Delta vehicle with no acceleration

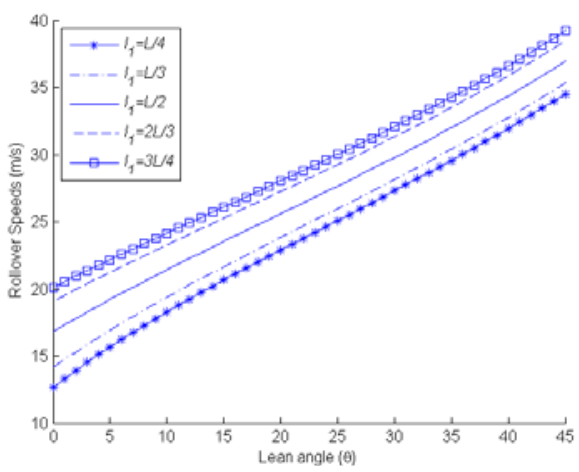

Figure 10. Three-wheeled Delta vehicle with acceleration 


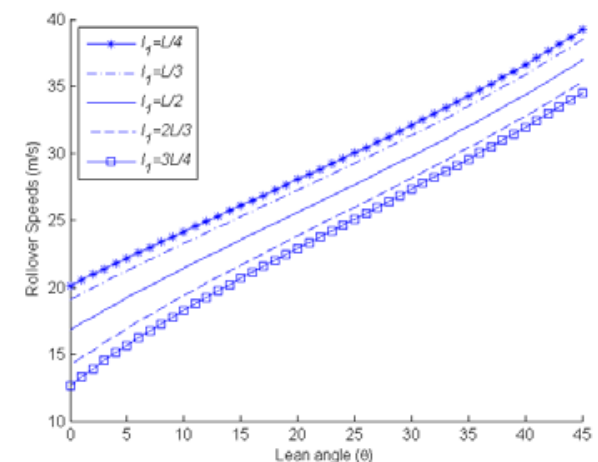

Figure 11. Three-wheeled Tadpole vehicle with deceleration

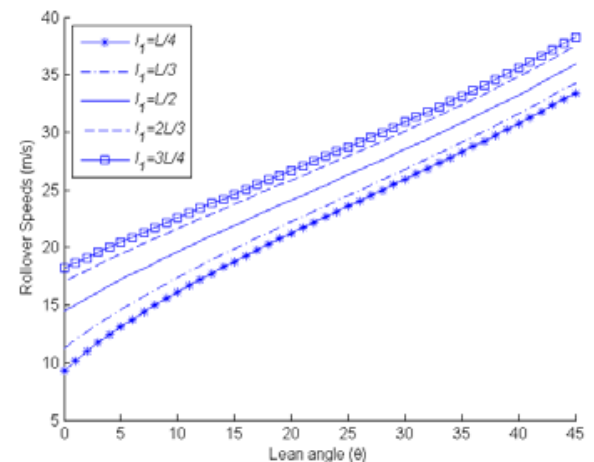

Figure 12. Three-wheeled Delta vehicle with deceleration

\section{Conclusion and discussion}

In this paper, the rollover stability conditions governing the tadpole and delta configurations of leaning three-wheeled vehicles have been presented and compared. From the above graphs, it is clear that leaning of the vehicle in a turn does increase the rollover stability for both the configuration of three wheeled vehicle. But, the maximum leaning angle will be limited by the vehicle design and, the friction between tire and road.

Furthermore from the graphs, it can be inferred that the vehicle has a better stability if the horizontal position of the centre of mass is close to the end with two wheels. In other words, the tadpole model is more stable if the centre of mass position is nearer to the front end and for the delta model, it should be nearer to the rear end. The mass of the passenger or the vehicle does not directly affect the rollover stability, but it affects the position of the centre of mass, which is indirectly going to change the stability in case of a three-wheeled vehicles. It can also be noted that the delta model is more stable while accelerating in a turn and the tadpole model is more stable while braking in a turn.

Thus, it can be concluded that by leaning the vehicle, the stability of a narrow three-wheeled vehicle could be matched with that of a standard four-wheeled vehicle. The outcome of this work can be useful for defining the optimal lean angle for a three-wheeled vehicle. Other factors like comfort of the passenger should also be considered while designing the optimal lean angle for a three-wheeled vehicle.

\section{References}

1. J.C. Huston, B.J. Graves and D.B. Johnson, "Three wheeled vehicle dynamics", SAE, Paper no. 820139 (1982)

2. Karnopp, D., Fang, C., "Simple model of steering-controlledbankingvehicles",ASME Dynamics Systems and Control Division (DSC), 44, Pg. 15-28 (1992).

3. Hibbard, R., Karnopp, D., "The dynamics of small, relativelytall and narrowtiltinggroundvehicles", ASME Dynamics Systems and Control Division (DSC), 52, Pg. 397-417 (1993).

4. Hibbard, R., Karnopp, D., "Methods of controlling the lean angle of tilting vehicles," ASME Dynamics Systems and Control Division (DSC), 52, Pg. 311-320(1993).

5. PalashPatodi, Vinay Saxena, YogeshRathore, "Review on tadpole design - Issues \& challenges", International Journal of Researchin Aeronautical and Mechanical Engineering, 2, Pg. 122-128 (2014).

6. Berote, Johan, Van Poelgeest, Auguste, Darling, Jos, Edge, Kevin, Plummer, Andrew, "The dynamics of a three-wheeled narrow-track tilting vehicle," University of Bath, F2008SC032. 